\title{
Mercury and cadmium trigger expression of the copper importer Ctr1B, which enables Drosophila to thrive on heavy metal-loaded food
}

\section{Kuppusamy Balamurugan, Haiqing Hua, Oleg Georgiev and Walter Schaffner*}

Institute of Molecular Biology, University of Zurich, Winterthurer Str. 190, CH-8057 Zurich, Switzerland

* Corresponding author

e-mail: walter.schaffner@molbio.uzh.ch

\begin{abstract}
Organisms from insects to mammals respond to heavy metal load (copper, zinc, cadmium, and mercury) by activating the metal-responsive transcription factor 1 (MTF1). MTF-1 binds to short DNA sequence motifs, termed metal response elements, and boosts transcription of a number of genes, notably those for metallothioneins. In Drosophila, MTF-1 somewhat counter-intuitively also activates transcription of a copper importer gene (Ctr1B) in response to copper starvation. Here, we report that mutant flies lacking Ctr1B are extremely sensitive to cadmium and mercury treatment, but can be rescued by excess copper in the food. We thus propose that copper, by competing for binding sites on cellular proteins, alleviates the toxic effects of mercury and cadmium. Such a scenario also explains a seemingly fortuitous metal response, namely, that cadmium and mercury strongly induce the expression of a Ctr1B reporter gene. Thus, the transcription enhancer/promoter region of the Ctr1B copper importer gene is subject to three modes of regulation. All of them depend on MTF-1 and all make biological sense, namely, (i) induction by copper starvation, (ii) repression by copper abundance, and (iii), as shown here, induction by cadmium or mercury at normal copper supply.
\end{abstract}

Keywords: copper transporter; heavy metal toxicity; metal homeostasis; MTF-1; non-essential metals.

Copper is an essential component of several important enzymes involved in cellular processes, such as respiration, oxidative stress protection, pigmentation, and iron homeostasis (Balamurugan and Schaffner, 2006; Kim et al., 2008). At the same time, copper poses a threat to the organism, especially as it can catalyze the generation of reactive oxygen species (ROS) via the Fenton reaction (Halliwell and Gutteridge, 1990; Puig and Thiele, 2002). Eukaryotic organisms from yeast to humans use elaborate systems to regulate copper homeostasis, consisting of copper importers, copper chaperones, inducible transcription factors, small metal binding proteins called metallothioneins, and copper exporters (O'Halloran and Culotta, 2000; Puig and Thiele, 2002; Mercer and Llanos, 2003; Rutherford and Bird, 2004; Balamurugan et al., 2007). High affinity copper transporters were identified in yeast (Ctr1, 2, and 3), mammals (Ctr1 and 2), and in Drosophila (Ctr1A, B, and C) (Dancis et al., 1994; Lee et al., 2000; Zhou et al., 2003). Null mutants of Ctr1 in the mouse and of Ctr1A in Drosophila are lethal, while a mutation of Drosophila Ctr1B results in a pigmentation defect and lethality under conditions of copper scarcity (Kuo et al., 2001; Lee et al., 2001; Zhou et al., 2003; Selvaraj et al., 2005; Turski and Thiele, 2007).

From insects to mammals, the major transcriptional regulator handling heavy metal toxicity is metal-responsive transcription factor 1 (MTF-1, also referred to as metal response element binding transcription factor 1) (Radtke et al., 1993; Otsuka et al., 2000; Andrews, 2001; Giedroc et al., 2001; Lichtlen and Schaffner, 2001; Egli et al., 2003; Balamurugan et al., 2004). MTF-1 is a zinc finger protein of some 700 amino acids that recognizes short DNA sequence motifs termed metal response elements (MREs) in the enhancer/promoter region of target genes, notably metallothionein genes (Stuart et al., 1984; Wang et al., 2004). MTF-1 is activated by heavy metal load and, somewhat counter-intuitively, also has a role during copper starvation, whereby it induces transcription of the Ctr1B copper importer in Drosophila (Selvaraj et al., 2005). Conversely, copper load results in an MTF1 dependent inhibition of Ctr1B expression (Zhou et al., 2003; Selvaraj et al., 2005). In follow-up experiments presented here we have made the observation that Ctr1B expression is indifferent to zinc status, but strongly induced by exposure of Drosophila larvae to the nonessential, toxic metals mercury and cadmium. What initially appeared as a fortuitous response to rare nonessential metals turned out to be of vital importance: flies lacking $C t r 1 B$ are extremely sensitive to mercury and cadmium, and toxicity can be alleviated by feeding the mutants a high-copper diet. Thus, Ctr1B counteracts mercury and cadmium toxicity by importing copper, which presumably acts as a less deleterious competitor for metal binding sites within the cell.

As a reporter gene to study expression and regulation of the Ctr1B copper importer, we used a Ctr1B transgene whose complete coding sequence was fused to the one of green fluorescent protein (eGFP) (Figure 1A). This construct, designated $\mathrm{AH} 3$, reproduced the regulation of the endogenous Ctr1B gene as determined by transcript mapping, namely, high expression upon copper starvation but low expression upon copper load (Zhou et al., 2003; Selvaraj et al., 2005). Similar to the endogenous 
A

AH3 copper importer/GFP fusion

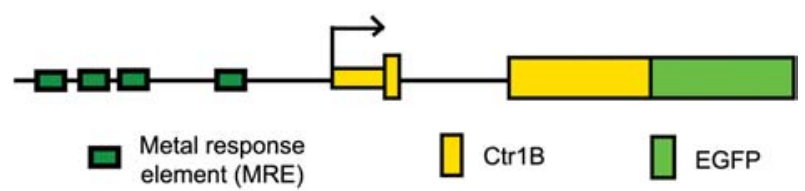

B

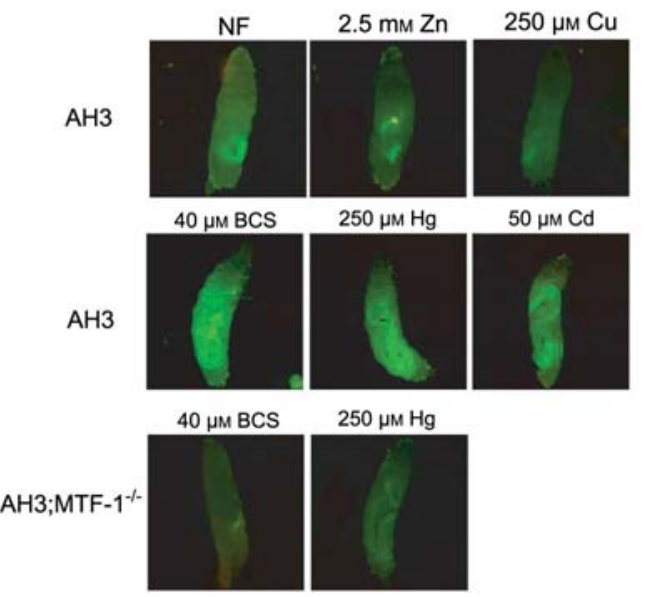

Figure 1 The Ctr1B copper importer gene is not only activated by copper starvation but also by the non-essential, toxic metals mercury and cadmium.

(A) Transgenic Ctr1B reporter gene ('AH3'; Selvaraj et al., 2005). In this construct, genomic DNA including the upstream enhancer with multiple metal response elements (MREs, shown as green boxes) and the complete $5^{\prime}$ UTR and coding sequence of Ctr1B is fused to the coding sequence of green fluorescent protein (eGFP). This indicator transgene, which still functions as a copper importer, was integrated via P-element transformation into the genome of Drosophila. (B) Expression of the Ctr1B reporter gene is activated by the non-essential metals mercury and cadmium. Wild-type flies or flies mutant for transcription factor MTF1 (dMTF-1140-1R; Egli et al., 2003) were allowed to lay eggs in culture tubes containing normal food (NF) or food with the indicated supplements. The typical intermediate level expression was not affected by zinc, but inhibited by copper load (first row). Ctr1B expression was induced by copper starvation (copper chelator BCS; Zhou et al., 2003; Selvaraj et al., 2005), and most notably also induced by cadmium or mercury ions (second row). This activation was dependent on the MRE-binding transcription factor MTF-1 (third row and not shown).

Methods: all fly strains used for this study were Oregon $\mathrm{R}$ with a yw background. Flies were grown from the egg stage in the indicated type of food (for simplicity, the terms or symbols copper, cadmium, zinc, and mercury refer to $\mathrm{Cu}^{2+}, \mathrm{Cd}^{2+}, \mathrm{Zn}^{2+}$, and $\mathrm{Hg}^{2+}$, respectively) and the resulting third instar larvae were taken for analysis. Images were taken with a Leica MZ FLIII fluorescence stereomicroscope and a Nikon Coolpix950 digital camera for whole larvae.

gene, regulated expression of this transgene depended on an upstream cluster of metal response elements (MREs) and on the presence of MTF-1, the cognate transcription factor: in MTF-1 null mutant larvae, expression remained low and was unaffected by copper concentration. In a complementary experiment we also included a treatment with other metals. While Ctr1B was indifferent to zinc supplement, expression was strongly induced by mercury or cadmium, two non-essential, notoriously toxic environmental pollutants (Figure 1B). Based on the fact that induction depended on MTF-1, we conclude
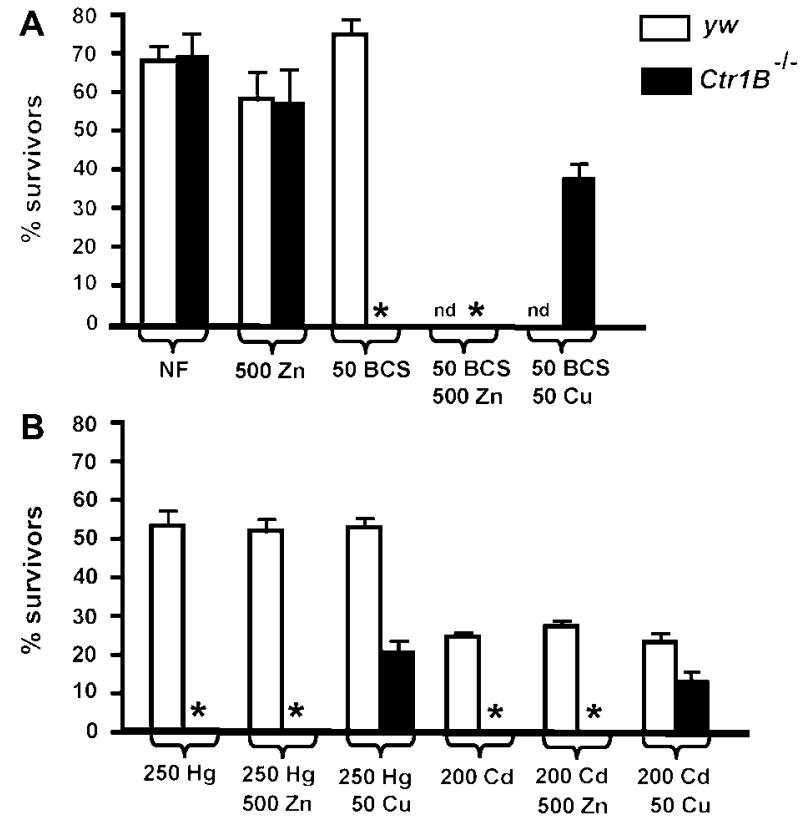

Figure 2 Sensitivity of Ctr1B mutants to cadmium and mercury is rescued by copper supplementation.

Ctr1B null mutants (Zhou et al., 2003) were compared to 'wild type' $(y w)$ flies. The percentage of laid eggs which develop to adulthood (enclosure from pupal stage) in the various types of food is indicated in the ordinate. (A) Control experiments. Most Drosophila eggs develop to adulthood in normal food (NF), with or without containing functional Ctr1B (white and black bars, respectively); mutants are killed by a moderate concentration of copper chelator, an effect which is rescued by copper, but not by zinc, supplement. Note that for simplicity all micromolar concentrations are indicated numerically (e.g., 500 $=500 \mu \mathrm{M}$ ). (B) Ctr1B null mutants are highly sensitive to mercury and cadmium but can be rescued to a large extent by copper supplement. Copper was supplemented simultaneously with other metals (cadmium, mercury). *Indicates no survivors; nd, not determined, but established previously to allow for survival of wild-type ( $y w)$ Drosophila (Egli et al., 2003 and data not shown).

that regulation of the Ctr1B copper importer gene also involves its upstream cluster of MREs, but with an effect opposite to that of copper. Initially, these results were interpreted as fortuitous response of fly larvae to toxic heavy metals which presumably are not regularly encountered in the wild. This view had to be revised when we also tested a Drosophila mutant lacking a functional Ctr1B. This fly strain develops well in normal food (Cu content, 2-5 $\mu \mathrm{M}$ ), but is sensitive to copper depletion. Unexpectedly, the Ctr1B mutant was also sensitive to the non-essential metals mercury and cadmium, while there was no sensitivity to zinc load. [Zinc homeostasis in higher eukaryotes including Drosophila is handled efficiently by a different set of transport proteins, notably ZIP-type importers and ZnT-type exporters (Mathews et al., 2005; Yepiskoposyan et al., 2006).] The sensitivity to cadmium and mercury was all the more surprising in light of the fact that Drosophila is quite metal-resistant and readily develops to adulthood in food containing either $200 \mu \mathrm{M}$ cadmium or $250 \mu \mathrm{M}$ mercury. Under these latter conditions, not a single larva of the Ctr1B mutant survived (Figure 2 and data not shown).

To test whether copper homeostasis was somehow linked to the ability of Drosophila to cope with cadmium 
Metallothionein regulation
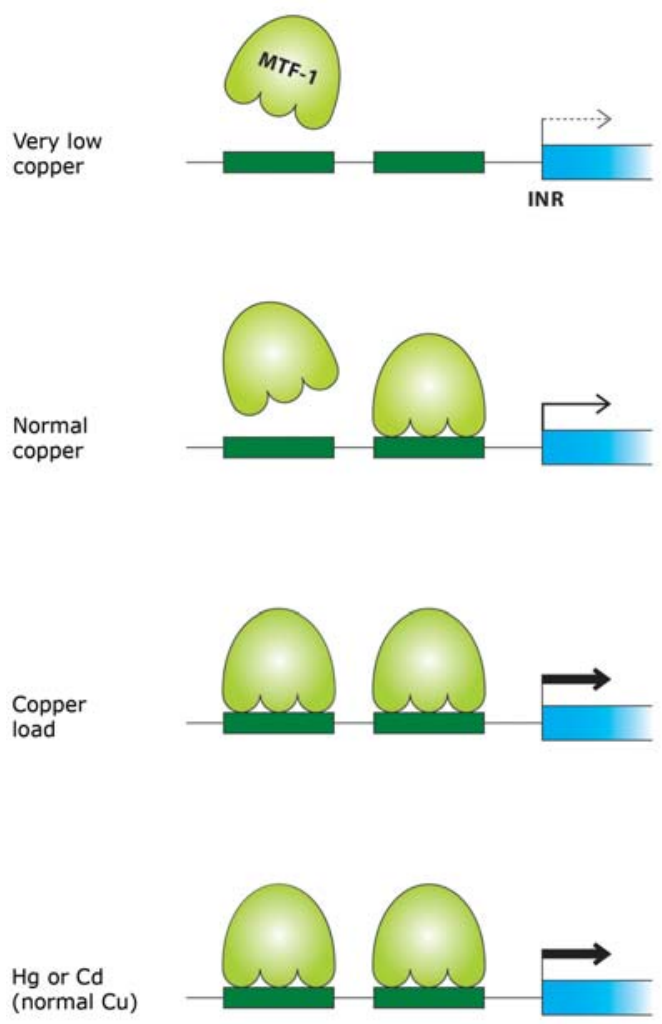
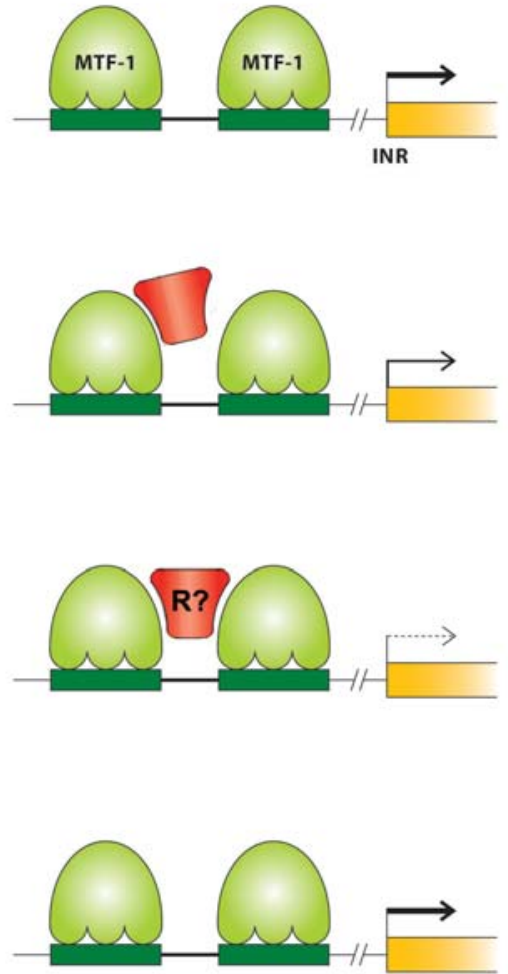

MRE

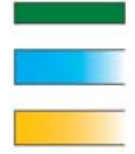

MT transcription unit

Ctr1B transcription unit

Figure 3 Model of differential response of metallothionein and Ctr1B genes to distortions of physiological metal concentrations. Transcriptional regulation of metallothionein and of Ctr1B copper importer genes is schematically shown on the left and right hand side, respectively. Regulation of both depends on metal-responsive transcription factor (MTF-1). Note that with copper, the two genes respond oppositely to abundance and depletion, indicated by thickness of arrows at the initiation region for transcription (INR): copper load has a negative effect on Ctr1B transcription by inducing an unfavorable MTF-1 conformation, mediated by the C-terminal cysteine cluster (Chen et al., 2008), or association with a (hypothetical, copper-specific) repressor (R?). The unique property of MTF-1-mediated repression is dependent on the specific spacing of metal response elements (MREs) (Selvaraj et al., 2005). By contrast, Ctr1B is induced, concordantly to metallothionein, by the toxic metals cadmium or mercury as a cellular defense reaction.

or mercury, we repeated the experiments whereby half of the food samples were also supplemented with $50 \mu \mathrm{M}$ copper salt. Indeed, while the sensitivity of the Ctr1B mutant to mercury and cadmium was reproduced, supplementation with copper largely rescued the lethality phenotype. By contrast, food supplementation with zinc failed to alleviate the deleterious effects of mercury or cadmium (Figure 2B). From these results, we conclude that copper plays a central role in counteracting the toxicity of the two non-essential metals. The extra copper imported by the larvae probably helps to fence off mercury and cadmium from binding to, and thereby inactivating, some essential protein(s). Alternatively, copper might exert its protective effect via a particularly strong and/or rapid induction of mRNAs encoding metallothioneins, the major heavy metal-scavenging proteins. We consider this possibility less likely, because both cadmium and mercury are potent inducers of metallothionein gene transcription (Zhang et al., 2001; Balamurugan et al., 2004; Egli et al., 2006). In any case, the seemingly awkward induction of Ctr1B upon treatment with mercury or cadmium (Figure 1B) is in fact biologically meaningful: there must be a sensing of the toxic effects and, mediated by MTF-1, a compensatory activation of the copper importer. It will be a challenge to find out why the upstream enhancer region of Ctr1B, including the MRE cluster, is repressed by copper, but responds to mercury and cadmium in a manner indistinguishable from metallothionein gene promoters, which are activated via MTF1 by a variety of metals, notably copper, zinc, cadmium, and mercury. One clue to this enigma is the spacing of metal response elements in the Ctr1B promoter. Spacing of three MREs is conserved among drosophilids and functionally important, while the MREs in metallothionein promoters mediate a metal response independently of their spacing. We have shown before that a synthetic regulatory sequence, consisting of the MREs of the Ctr1B upstream enhancer but with reduced spacing 
between them, behaves like a metallothionein promoter in that it becomes responsive to copper, instead of being repressed (Selvaraj et al., 2005). It is tempting to speculate that the combination of MREs and their spacing is generating a situation where copper, unlike mercury and cadmium, induces an unfavorable conformation of MTF1 or a recruitment of a (hypothetical) repressor to the regulatory DNA of the Ctr1B gene (see model in Figure 3).

What originally appeared to be an erratic response to toxic agents, which might rarely be a threat to wild-living Drosophila, turned out to be a biologically meaningful, life-saving response. This response is further testimony to a surprising ability of living systems to cope with situations which are not part of their daily encountered, perhaps not even once-per-generation encountered, environmental conditions.

\section{Acknowledgments}

We are grateful to Antonia Manova for technical assistance, Till Strassen for maintaining the fly stocks, Dominik Steiger for valuable discussions, George Hausmannn for critical reading of the manuscript and Erika Schaffner for help in preparing the figures. This work was supported by the Swiss National Science Foundation and the Kanton Zürich.

\section{References}

Andrews, G.K. (2001). Cellular zinc sensors: MTF-1 regulation of gene expression. Biometals 14, 223-237.

Balamurugan, K. and Schaffner, W. (2006). Copper homeostasis in eukaryotes: teetering on a tightrope. Biochim. Biophys. Acta 1763, 737-746.

Balamurugan, K., Egli, D., Selvaraj, A., Zhang, B., Georgiev, O., and Schaffner, W. (2004). Metal-responsive transcription factor (MTF-1) and heavy metal stress response in Drosophila and mammalian cells: a functional comparison. Biol. Chem. 385, 597-603.

Balamurugan, K., Egli, D., Hua, H., Rajaram, R., Seisenbacher, G., Georgiev, O., and Schaffner, W. (2007). Copper homeostasis in Drosophila by complex interplay of import, storage and behavioral avoidance. EMBO J. 26, 1035-1044

Chen, X., Hua, H., Balamurugan, K., Kong, X., Zhang, L., George, G.N., Georgiev, O., Schaffner, W., and Giedroc, D.P. (2008). Copper sensing function of Drosophila metal-responsive transcription factor- 1 is mediated by a tetranuclear $\mathrm{Cu}(\mathrm{l})$ cluster. Nucleic Acids Res. 36, 3128-3138.

Dancis, A., Yuan, D.S., Haile, D., Askwith, C., Eide, D., Moehle, C., Kaplan, J., and Klausner, R. (1994). Molecular characterization of a copper transport protein in S. cerevisiae: an unexpected role for copper in iron transport. Cell 76, 393-402.

Egli, D., Selvaraj, A., Yepiskoposyan, H., Zhang, B., Hafen, E., Georgiev, O., and Schaffner, W. (2003). Knockout of 'metalresponsive transcription factor' MTF-1 in Drosophila by homologous recombination reveals its central role in heavy metal homeostasis. EMBO J. 22, 100-108.

Egli, D., Yepiskoposyan, H., Selvaraj, A., Balamurugan, K., Rajaram, R., Simons, A., Multhaup, G., Mettler, S., Vardanyan, A., Georgiev, O., et al. (2006). A family knockout of all four Drosophila metallothioneins reveals a central role in copper homeostasis and detoxification. Mol. Cell. Biol. 26, 2286-2296.
Giedroc, D.P., Chen, X., and Apuy, J.L. (2001). Metal response element (MRE)-binding transcription factor-1 (MTF-1): structure, function, and regulation. Antiox. Redox Signal. 3, 577596.

Halliwell, B. and Gutteridge, J.M. (1990). Role of free radicals and catalytic metal ions in human disease: an overview. Methods Enzymol. 186, 1-85.

Kim, B.E., Nevitt, T., and Thiele, D.J. (2008). Mechanisms for copper acquisition, distribution and regulation. Nat. Chem. Biol. 4, 176-185.

Kuo, Y.M., Zhou, B., Cosco, D., and Gitschier, J. (2001). The copper transporter CTR1 provides an essential function in mammalian embryonic development. Proc. Natl. Acad. Sci. USA 98, 6836-6841.

Lee, J., Prohaska, J.R., Dagenais, S.L., Glover, T.W., and Thiele, D.J. (2000). Isolation of a murine copper transporter gene, tissue specific expression and functional complementation of a yeast copper transport mutant. Gene 254, 87-96.

Lee, J., Prohaska, J.R., and Thiele, D.J. (2001). Essential role for mammalian copper transporter Ctr1 in copper homeostasis and embryonic development. Proc. Natl. Acad. Sci. USA 98, 6842-6847.

Lichtlen, P. and Schaffner, W. (2001). Putting its fingers on stressful situations: the heavy metal-regulatory transcription factor MTF-1. Bioessays 23, 1010-1017.

Mathews, W.R., Wang, F., Eide, D.J., and Van Doren, M. (2005). Drosophila fear of intimacy encodes a Zrt/IRT-like protein (ZIP) family zinc transporter functionally related to mammalian ZIP proteins. J. Biol. Chem. 280, 787-795.

Mercer, J.F. and Llanos, R.M. (2003). Molecular and cellular aspects of copper transport in developing mammals. J. Nutr. 133 (5 Suppl. 1), 1481S-1484S.

O'Halloran, T.V. and Culotta, V.C. (2000). Metallochaperones, an intracellular shuttle service for metal ions. J. Biol. Chem. 275, 25057-25060.

Otsuka, F., Okugaito, I., Ohsawa, M., Iwamatsu, A., Suzuki, K., and Koizumi, S. (2000). Novel responses of ZRF, a variant of human MTF-1, to in vivo treatment with heavy metals. Biochim. Biophys. Acta 1492, 330-340.

Puig, S. and Thiele, D.J. (2002). Molecular mechanisms of copper uptake and distribution. Curr. Opin. Chem. Biol. 6, $171-180$

Radtke, F., Heuchel, R., Georgiev, O., Hergersberg, M., Gariglio, M., Dembic, Z., and Schaffner, W. (1993). Cloned transcription factor MTF-1 activates the mouse metallothionein I promoter. EMBO J. 12, 1355-1362.

Rutherford, J.C. and Bird, A.J. (2004). Metal-responsive transcription factors that regulate iron, zinc, and copper homeostasis in eukaryotic cells. Eukaryot. Cell 3, 1-13.

Selvaraj, A., Balamurugan, K., Yepiskoposyan, H., Zhou, H., Egli, D., Georgiev, O., Thiele, D.J., and Schaffner, W. (2005). Metalresponsive transcription factor (MTF-1) handles both extremes, copper load and copper starvation, by activating different genes. Genes Dev. 19, 891-896.

Stuart, G.W., Searle, P.F., Chen, H.Y., Brinster, R.L., and Palmiter, R.D. (1984). A 12-base-pair DNA motif that is repeated several times in metallothionein gene promoters confers metal regulation to a heterologous gene. Proc. Natl. Acad. Sci. USA $81,7318-7322$.

Turski, M.L. and Thiele, D.J. (2007). Drosophila Ctr1A functions as a copper transporter essential for development. J. Biol. Chem. 282, 24017-24026.

Wang, Y., Lorenzi, I., Georgiev, O., and Schaffner, W. (2004). Metal-responsive transcription factor-1 (MTF-1) selects different types of metal response elements at low vs. high zinc concentration. Biol. Chem. 385, 623-632.

Yepiskoposyan, H., Egli, D., Fergestad, T., Selvaraj, A., Treiber, C., Multhaup, G., Georgiev, O., and Schaffner, W. (2006). Transcriptome response to heavy metal stress in Drosophila 
reveals a new zinc transporter that confers resistance to zinc. Nucleic Acids Res. 34, 4866-4877.

Zhang, B., Egli, D., Georgiev, O., and Schaffner, W. (2001). The Drosophila homolog of mammalian zinc finger factor MTF-1 activates transcription in response to heavy metals. Mol. Cell. Biol. 21, 4505-4514.
Zhou, H., Cadigan, K.M., and Thiele, D.J. (2003). A copper-regulated transporter required for copper acquisition, pigmentation, and specific stages of development in Drosophila melanogaster. J. Biol. Chem. 278, 48210-48218.

Received September 19, 2008; accepted November 4, 2008 